VOL. 68 (2003) [517-528]

\title{
A SUPERCONVERGENCE RESULT FOR SOLUTIONS OF COMPACT OPERATOR EQUATIONS
}

\author{
REKHA P. KULKARNI
}

\begin{abstract}
Over the last 20 years, since the publication of Sloan's paper on the improvement by the iteration technique, various approaches have been proposed for post-processing the Galerkin solution of multi-dimensional second kind Fredholm Integral equation. These methods include the iterated Galerkin method proposed by Sloan, the Kantorovich method and the iterated Kantorovich method. Recently, Lin, Zhang and Yan have proposed interpolation as an alternative to the iteration technique. For an integral operator, with a smooth kernel using the orthogonal projection onto a space of discontinuous piecewise polynomials of degree $\leqslant r-1$, previous authors have established an order $r$ convergence for the Galerkin solution and $2 r$ for the iterated Galerkin solution. Equivalent results have also been established for the interpolatory projection at Gauss points and some interpolation post-processing technique. In this paper, a method is introduced and shown to have convergence of order $4 r$. The size of the system of equations that must be solved, in implementing this method, remains the same as for the Galerkin method.
\end{abstract}

\section{INTRODUCTION}

The improvement of the Galerkin solution of a compact operator equation by using iteration techniques was first proposed by Sloan in [13]. Chandler [1], in his thesis, proved that if the kernel and the exact solution are suitably smooth, then, in the case of the orthogonal projection onto a space of piecewise polynomials, the order of convergence of the iterated Galerkin solution is twice that of the Galerkin solution. He also established that, if the kernel fails to have sufficient differentiability because of discontinuities along the diagonal, then the iterated Galerkin solution is only superconvergent at the knot points, not globally. Similar results for the iterated collocation at Gauss points are given in Chatelin and Lebbar [3]. Chandler [1] also has discussed the superconvergent behaviour of the discrete iterated Galerkin solution.

Received 14th July, 2003

This work was partly done during the visit to CMA (MSI), Australian National University and CSIRO, Mathematical and Information Sciences, Canberra, Australia. The author wishes to thank Markus Hegland for fruitful discussions and Bob Anderssen for his encouragement, for reading the manuscript carefully and making valuable suggestions.

Copyright Clearance Centre, Inc. Serial-fee code: 0004-9727/03 \$A2.00+0.00. 
If the right hand side of the operator equation is less smooth than the kernel of the integral operator, then the Kantorovich solution has a higher order of convergence than the Galerkin solution. This is discussed in Schock [12] and Sloan [14]. Sloan also introduced the iterated Kantorovich method and established that it had a faster convergence than the Galerkin, the iterated Galerkin and the Kantorovich methods. Graham, Joe and Sloan [7] have compared the iterated Galerkin and the iterated collocation methods under general smoothness requirements. An unified framework for error analysis of the Galerkin and the discrete Galerkin methods using prolongation and restriction operators is developed by Spence and Thomas in [15]. More recently, Lin, Zhang and Yan [10] have proposed interpolation post-processing technique as an alternative to the iteration technique for improving collocation solution. They obtain the same order of convergence as for the iterated collocation method. In [8], $\mathrm{Hu}$ discusses interpolation post-processing technique for Fredholm Integro-differential equations.

In this paper, we propose a method based on projections for approximate solutions of compact operator equations. It is shown that, while it is necessary to solve a system of equations of the same size as for the Galerkin method, the resulting solution obtained converges faster than the Galerkin and the iterated Galerkin solutions. As the only assumptions required are compactness of the operator and the pointwise convergence of the projection operators, the method can be applied to integral operators with continuous kernels defined on curves or surfaces. For both orthogonal projections and the interpolatory projections at Gauss points with the range as a space of piecewise polynomials, it is established that, if the kernel and the right hand side are suitably smooth, then the order of convergence in the iterated version of the proposed method, is twice that of the iterated Galerkin solution and four times that of the Galerkin solution.

In the proposed method, it is necessary to solve a different system of equations than in the Galerkin method. Hence, in contrast to the iteration technique of Sloan and the interpolation technique of Lin, Zhang, Yan and $\mathrm{Hu}$, the proposed method cannot be considered as a post-processing method for Galerkin solutions. In fact, a new sequence of finite rank operators converging in norm is proposed. As a direct consequence, as in Kulkarni [9], this method can also be used to solve eigenvalue problems. The methodology and techniques, introduced in this paper, extend naturally to iterative refinement schemes, multilevel methods and extrapolation.

The paper has been arranged in the following way. In Section 2, the proposed method for the solution of compact operator equations is defined along with relevant notation. In Section 3, error estimates are derived in a general setting. The system of linear equations which need to be solved to obtain the approximations to the solution of the operator equation is also discussed. The special situation, where the compact operator is an integral operator with a smooth kernel and the projection is either the orthogonal projection or the interpolatory projection, is considered in Section 4. Precise 
orders of convergence are also obtained there. Numerical validation is given in Section 5 .

\section{Method, Notation and Definitions}

Let

$$
u-T u=f
$$

denote a second kind operator equation, where $T$ is a compact linear operator defined on a complex Banach space $X$ and $f$ and $u$ belong to $X$. We assume that $(I-T)$ is invertible, so that (2.1) has a unique solution. A standard technique to solve (2.1) approximately is to replace $T$ by a finite rank operator. The approximate solution of (2.1) is then obtained by essentially solving a system of linear equations. Let $\pi_{n}$ be a sequence of projection operators converging to the identity operator $I$ pointwise. In the classical Galerkin method $T$ is replaced by $T_{n}^{G}=\pi_{n} T \pi_{n}$ and the right hand side $f$ is replaced by $\pi_{n} f$. In the iterated Galerkin method proposed by Sloan, $T$ is replaced by $T_{n}^{S}=T \pi_{n}$, and in the Kantorovich method $T$ is replaced by $T_{n}^{K}=\pi_{n} T$.

Here, we propose to approximate $T$ by the following finite rank operator

$$
T_{n}^{M}=\pi_{n} T \pi_{n}+\pi_{n} T\left(I-\pi_{n}\right)+\left(I-\pi_{n}\right) T \pi_{n} .
$$

Then

$$
\left\|T-T_{n}^{M}\right\|=\left\|\left(I-\pi_{n}\right) T\left(I-\pi_{n}\right)\right\| \rightarrow 0 \text { as } n \rightarrow \infty .
$$

The corresponding approximation of $(2.1)$ becomes

$$
u_{n}^{M}-\left(\pi_{n} T \pi_{n}+\pi_{n} T\left(I-\pi_{n}\right)+\left(I-\pi_{n}\right) T \pi_{n}\right) u_{n}^{M}=f,
$$

while the iterative refinement is defined by

$$
\widetilde{u}_{n}^{M}=T u_{n}^{M}+f .
$$

It is shown that under certain conditions $u_{n}^{M}$ converges to $u$ faster than the approximations obtained by the Galerkin and Sloan methods and that $\widetilde{u}_{n}^{M}$ converges faster than $u_{n}^{M}$.

Consider the integral operator

$$
(T x)(s)=\int_{0}^{1} k(s, t) x(t) d m(t), \quad s \in[0,1]
$$

where the kernel $k(.,.) \in C([0,1] \times[0,1])$. Then $T: L^{2}[0,1] \rightarrow L^{2}[0,1]$ or $T: C[0,1]$ $\rightarrow C[0,1]$ is a compact linear operator. Let $r \geqslant 1$. If $k(.,.) \in C^{r}([0,1] \times[0,1])$, then 
$R(T) \subset C^{r}[0,1]$. Thus, if $k(.,.) \in C^{r}([0,1] \times[0,1])$ and $f \in C^{r}[0,1]$, then $u \in C^{r}[0,1]$. For $u \in C^{r}[0,1]$, let $u^{(r)}$ denote the $r$-th derivative of $u$. We set

$$
\begin{aligned}
D^{i, j} k(s, t) & =\frac{\partial^{i+j}}{\partial s^{i} \partial t^{j}} k(s, t), \quad s, t \in[0,1], \\
\|k\|_{r, \infty} & =\sum_{i=0}^{r} \sum_{j=0}^{r}\left\|D^{i, j} k\right\|_{\infty}
\end{aligned}
$$

and

$$
\|u\|_{r, \infty}=\sum_{i=0}^{r}\left\|u^{(i)}\right\|_{\infty}
$$

For any integer $n$, let

$$
0=t_{0}<t_{1}<\cdots<t_{n}=1
$$

be a partition of $[0,1]$ and for $i=1, \ldots, n$, set $h_{i}=t_{i}-t_{i-1}, h=\max _{i} h_{i}$. It is assumed that $h \rightarrow 0$ as $n \rightarrow \infty$. Let $X_{n}=S_{r, n}^{\nu}$, the space of all piecewise polynomials of order $r$ (that is, of degree $\leqslant r-1$ ) with breakpoints at $t_{1}, \ldots, t_{n-1}$ and with $\nu$ continuous derivatives, $-1 \leqslant \nu \leqslant r-2$. Here $\nu=0$ corresponds to the case of continuous piecewise polynomials. If $\nu=-1$, there is no continuity requirements at the breakpoints, in which case $\phi_{n} \in X_{n}$ is arbitrarily taken to be left continuous at $t_{1}, \ldots, t_{n}$ and right continuous at $t_{0}$.

\section{ERror Estimates}

In this Section, it is assumed that $T$ is a compact operator and $\pi_{n}$ is a sequence of projections converging to the identity operator pointwise. The error estimates for $u_{n}^{M}$ and $\widetilde{u}_{n}^{M}$ are obtained below.

THEOREM 3.1. For all large $n$,

$$
\left\|u-u_{n}^{M}\right\| \leqslant C_{1}\left\|\left(I-\pi_{n}\right) T\left(I-\pi_{n}\right) u\right\|
$$

and

$$
\begin{aligned}
&\left\|u-\widetilde{u}_{n}^{M}\right\| \leqslant\left\|(I-T)^{-1}\right\|\left(\left\|T\left(I-\pi_{n}\right) T\left(I-\pi_{n}\right) u\right\|\right. \\
&+\left.\left\|T\left(I-\pi_{n}\right) T\left(I-\pi_{n}\right)\right\|\left\|u-u_{n}^{M}\right\|\right),
\end{aligned}
$$

where $C_{1}$ is a constant independent of $n$.

PROOF: Since $\left\|T-T_{n}^{M}\right\| \rightarrow 0$ as $n \rightarrow \infty$, for all large $n,\left(I-T_{n}^{M}\right)$ is invertible and $\left\|\left(I-T_{n}^{M}\right)^{-1}\right\| \leqslant C_{1}$, a constant independent of $n$. 
We have

$$
\begin{aligned}
u-u_{n}^{M} & =\left[(I-T)^{-1}-\left(I-T_{n}^{M}\right)^{-1}\right] f \\
& =\left(I-T_{n}^{M}\right)^{-1}\left(T-T_{n}^{M}\right) u
\end{aligned}
$$

Thus,

$$
\begin{aligned}
\left\|u-u_{n}^{M}\right\| & \leqslant\left\|\left(I-T_{n}^{M}\right)^{-1}\right\|\left\|\left(I-\pi_{n}\right) T\left(I-\pi_{n}\right) u\right\| \\
& \leqslant C_{1}\left\|\left(I-\pi_{n}\right) T\left(I-\pi_{n}\right) u\right\|,
\end{aligned}
$$

which completes the proof of (3.1).

Also, since

$$
\begin{aligned}
u-\widetilde{u}_{n}^{M} & =T\left(u-u_{n}^{M}\right) \\
& =T(I-T)^{-1}\left(T-T_{n}^{M}\right)\left(I-T_{n}^{M}\right)^{-1} f \\
& =(I-T)^{-1} T\left(I-\pi_{n}\right) T\left(I-\pi_{n}\right)\left(u+u_{n}^{M}-u\right)
\end{aligned}
$$

(3.2) follows.

Let $u_{n}^{G}$ and $u_{n}^{S}$ be the approximate solutions of operator equation (2.1) using Galerkin and Sloan methods, respectively. Then

$$
\begin{aligned}
u_{n}^{G}-\pi_{n} T \pi_{n} u_{n}^{G} & =\pi_{n} f \\
u_{n}^{S}-T \pi_{n} u_{n}^{S} & =f .
\end{aligned}
$$

We quote the following error estimates from Theorem 2.1 of $([14])$.

$$
\begin{aligned}
& \left\|u-u_{n}^{G}\right\| \leqslant C_{1}\left\|\left(I-\pi_{n}\right) u\right\|, \\
& \left\|u-u_{n}^{S}\right\| \leqslant C_{1}\left\|T\left(I-\pi_{n}\right) u\right\| \leqslant C_{1}\left\|T\left(I-\pi_{n}\right)\right\|\left\|u-u_{n}^{G}\right\| .
\end{aligned}
$$

Thus it is clear that $u_{n}^{M}$ is more accurate than $u_{n}^{G}$ and $u_{n}^{S}$.

In the next section, the precise orders of convergence are obtained in the case when $T$ is an integral operator and $\pi_{n}$ is either the orthogonal projection or the interpolatory projection at Gauss points.

3.1. Computational Cost. The reduction of (2.2) to a system of linear equations is now considered.

Applying $\pi_{n}$ and $\left(I-\pi_{n}\right)$ to equation (2.2) we obtain

$$
\begin{aligned}
\pi_{n} u_{n}^{M}-\pi_{n} T \pi_{n} u_{n}^{M}-\pi_{n} T\left(I-\pi_{n}\right) u_{n}^{M} & =\pi_{n} f, \\
\left(I-\pi_{n}\right) u_{n}^{M}-\left(I-\pi_{n}\right) T \pi_{n} u_{n}^{M} & =\left(I-\pi_{n}\right) f .
\end{aligned}
$$

Let $w_{n}^{M}=\pi_{n} u_{n}^{M}$. The substitution for $\left(I-\pi_{n}\right) u_{n}^{M}$ from equation (3.6) in equation (3.5) gives us

$$
w_{n}^{M}-\left(\pi_{n} T \pi_{n}+\pi_{n} T\left(I-\pi_{n}\right) T \pi_{n}\right) w_{n}^{M}=\pi_{n} f+\pi_{n} T\left(I-\pi_{n}\right) f
$$


We then have

$$
u_{n}^{M}=w_{n}^{M}+\left(I-\pi_{n}\right) T w_{n}^{M}+\left(I-\pi_{n}\right) f
$$

and

$$
\tilde{u}_{n}^{M}=f+T u_{n}^{M}
$$

If the dimension of $R\left(\pi_{n}\right)$ is $n_{1}$, then (3.7) reduces to a system of equations of size $n_{1}$. Thus, a system of the same size as in the case of Galerkin method is required to be solved. The extra cost in the method proposed here is the construction of the matrix corresponding to $\pi_{n} T\left(I-\pi_{n}\right) T \pi_{n}$ and computation of the vectors $\pi_{n} T\left(I-\pi_{n}\right) f$ and $T w_{n}^{M}$. This addition in the cost is compensated by the improvement in the order of the convergence.

\section{Orders of Convergence}

4.1. Orthogonal Projection. Let $X=L^{2}[0,1]$ and $\langle$,$\rangle denotes the usual inner$ product on $X$. Let $T$ be an integral operator defined by (2.4) with a kernel $k(\cdot, \cdot)$ $\in C^{r}([0,1]) \times[0,1]$. Let $X_{n}=S_{r, n}^{\nu}$ and $\pi_{n}: X \rightarrow X_{n}$ denote the orthogonal projection. When $\nu=-1$ or 0 it is known, without any restriction on the partition, that (see Richter ([11]) and de Boor([4]), respectively)

$$
\left\|\pi_{n}\right\|_{L^{\infty} \rightarrow L^{\infty}} \leqslant c
$$

When $\nu \geqslant 1,(4.1)$ remains true with the additional restriction that the partition is quasiuniform (see Douglas, Dupont and Wahlbin [6]).

Since $\pi_{n} y \rightarrow y$ as $n \rightarrow \infty$ for each $y \in X$, the results of Section 3 are applicable. In what follows the following estimate is crucially used.

For $y \in C^{r}[0,1]$, (see Chatelin [2], Corollary 7.6, p. 328),

$$
\left\|\left(I-\pi_{n}\right) y\right\|_{\infty} \leqslant C_{1}\left\|y^{(r)}\right\|_{\infty} h^{r}
$$

(In this paper $C_{1}, C_{2}$, and $C_{3}$ denote generic constants, which may take different values at their different occurences, but will be independent of $n$ and of $h$.)

A preliminary result using standard techniques is proved below.

Proposition 4.1. For $y \in C^{r}[0,1]$, we have

$$
\left\|T\left(I-\pi_{n}\right) y\right\|_{r, \infty} \leqslant\left(C_{1}\right)^{2}(r+1)\|k\|_{r, \infty}\left\|y^{(r)}\right\|_{\infty} h^{2 r} .
$$

Proof: For a fixed $j$ such that $0 \leqslant j \leqslant r$, we have

$$
\left[T\left(I-\pi_{n}\right) y\right]^{(j)}(s)=\int_{0}^{l} \frac{\partial^{j}}{\partial s^{j}} k(s, t)\left(I-\pi_{n}\right) y(t) d t
$$


Let

$$
\ell(s, t)=\frac{\partial^{j}}{\partial s^{j}} k(s, t), s, t \in[0,1] .
$$

For a fixed $s \in[0,1]$, we denote $\ell_{s}(t)=\ell(s, t), t \in[0,1]$ and $\bar{\ell}_{s}$ denotes the complex conjugate of $\ell_{s}$. With this notation,

$$
\begin{aligned}
{\left[T\left(I-\pi_{n}\right) y\right]^{(j)}(s) } & =\left\langle\left(I-\pi_{n}\right) y, \overline{\ell_{s}}\right\rangle \\
& =\left\langle\left(I-\pi_{n}\right) y,\left(I-\pi_{n}\right) \bar{\ell}_{s}\right\rangle,
\end{aligned}
$$

since $\pi_{n}$ is an orthogonal projection. Thus for each $s \in[0,1]$, we have

$$
\begin{aligned}
\left|\left[T\left(I-\pi_{n}\right) y\right]^{(j)}(s)\right| & \leqslant\left\|\left(I-\pi_{n}\right) y\right\|_{\infty}\left\|\left(I-\pi_{n}\right) \bar{\ell}_{s}\right\|_{\infty} \\
& \leqslant\left(C_{1}\right)^{2}\left\|y^{(r)}\right\|_{\infty}\left\|\left(\bar{\ell}_{s}\right)^{(r)}\right\|_{\infty} h^{2 r} \\
& \leqslant\left(C_{1}\right)^{2}\left\|y^{(r)}\right\|_{\infty}\|k\|_{r, \infty} h^{2 r} .
\end{aligned}
$$

Hence taking supremum over $s \in[0,1]$, we obtain

$$
\left\|\left[T\left(I-\pi_{n}\right) y\right]^{(j)}\right\|_{\infty} \leqslant\left(C_{1}\right)^{2}\|k\|_{r, \infty}\left\|y^{(r)}\right\|_{\infty} h^{2 r}
$$

and

$$
\left\|T\left(I-\pi_{n}\right) y\right\|_{r, \infty}=\sum_{j=0}^{r}\left\|\left[T\left(I-\pi_{n}\right) y\right]^{(j)}\right\|_{\infty} \leqslant\left(C_{1}\right)^{2}(r+1)\|k\|_{r, \infty}\left\|y^{(r)}\right\|_{\infty} h^{2 r} .
$$

Estimates for terms appearing in (3.1) and (3.2) are now obtained.

Proposition 4.2. For $y \in C^{r}[0,1]$, we have

$$
\left\|\left(I-\pi_{n}\right) T\left(I-\pi_{n}\right) y\right\|_{\infty} \leqslant C_{2} h^{3 r},
$$

and

$$
\left\|T\left(I-\pi_{n}\right) T\left(I-\pi_{n}\right) y\right\|_{\infty} \leqslant C_{3} h^{4 r} .
$$

Proof: By the estimate (4.2) we get

$$
\left\|\left(I-\pi_{n}\right) T\left(I-\pi_{n}\right) y\right\|_{\infty} \leqslant C_{1}\left\|\left[T\left(I-\pi_{n}\right) y\right]^{(r)}\right\|_{\infty} h^{r} .
$$

Using the estimate (4.4) we get

$$
\left\|\left(I-\pi_{n}\right) T\left(I-\pi_{n}\right) y\right\|_{\infty} \leqslant\left(C_{1}\right)^{3}\|k\|_{r, \infty}\left\|y^{(r)}\right\|_{\infty} h^{3 r},
$$

which completes the proof of (4.5), with $C_{2}=\left(C_{1}\right)^{3}\|k\|_{r, \infty}\left\|y^{(r)}\right\|_{\infty}$.

Next using (4.4) we obtain

$$
\begin{aligned}
\left\|T\left(I-\pi_{n}\right) T\left(I-\pi_{n}\right) y\right\|_{\infty} & \leqslant\left(C_{1}\right)^{2}\|k\|_{r, \infty}\left\|\left(T\left(I-\pi_{n}\right) y\right)^{(r)}\right\|_{\infty} h^{2 r} \\
& \leqslant\left(\left(C_{1}\right)^{2}\|k\|_{r, \infty}\right)^{2}\left\|y^{(r)}\right\|_{\infty} h^{4 r},
\end{aligned}
$$

which completes the proof of (4.6), with $C_{3}=\left(\left(C_{1}\right)^{2}\|k\|_{r, \infty}\right)^{2}\left\|y^{(r)}\right\|_{\infty}$. 
4.2. Interpolatory Projection. Let $X=C[0,1]$ with the supremum norm. Choose $r \geqslant 1$ and let $k(.,.) \in C^{2 r}([0,1] \times[0,1])$. Then the integral operator defined by (2.4) is a compact linear operator on $C[0,1]$.

Let $X_{n}=S_{r, n}^{-1}$, the space of all piecewise polynomials of order $r$ with breakpoints at $t_{1}, \ldots, t_{n-1}$.

Let $B_{r}=\left\{\tau_{1}, \ldots, \tau_{r}\right\}$ denote the set of $r$ Gauss points, that is, the zeros of the (Legendre) polynomial $\left(d^{r} / d s^{r}\right)\left(s^{2}-1\right)^{r}$ in the interval $[-1,1]$. Define $f_{i}:[-1,1]$ $\rightarrow\left[t_{i-1}, t_{i}\right]$ as follows.

$$
f_{i}(t)=\frac{1-t}{2} t_{i-1}+\frac{1+t}{2} t_{i}, \quad t \in[-1,1] .
$$

Let $A=\bigcup_{i=1}^{n} f_{i}\left(B_{r}\right)=\left\{\tau_{i j}=f_{i}\left(\tau_{j}\right): 1 \leqslant i \leqslant n, 1 \leqslant j \leqslant r\right\}$, the set of $n r$ Gauss points. The operator $\pi_{n}: C[0,1] \rightarrow X_{n}$ is defined by

$$
\pi_{n} u \in X_{n},\left(\pi_{n} u\right)\left(\tau_{i j}\right)=u\left(\tau_{i j}\right), 1 \leqslant i \leqslant n, 1 \leqslant j \leqslant r .
$$

Then $\pi_{n} u \rightarrow u$ as $n \rightarrow \infty$ for each $u \in C[0,1]$ and the results of Section 2 are applicable.

We quote the following two estimates.

For $u \in C^{r}[0,1]$, (See Chatelin [2], Corollary 7.6, p. 328),

$$
\left\|\left(I-\pi_{n}\right) u\right\|_{\infty} \leqslant C_{1}\left\|u^{(r)}\right\|_{\infty} h^{r}
$$

Let $f \in C^{r}[0,1]$ and $g \in C^{2 r}[0,1]$. Then, (See de-Boor-Swartz [5]),

$$
\left|\int_{0}^{1} f(t)\left(I-\pi_{n}\right) g(t) d t\right| \leqslant C_{2}\|f\|_{r, \infty}\|g\|_{2 r, \infty} h^{2 r} .
$$

As the proofs of the following two Propositions follow in a straightforward manner by using the above two estimates, the results are only stated.

Proposition 4.3. For $y \in C^{2 r}[0,1]$, we have

$$
\left\|T\left(I-\pi_{n}\right) y\right\|_{2 r, \infty} \leqslant C_{2}(2 r+1)\|k\|_{2 r, \infty}\|y\|_{2 r, \infty} h^{2 r},
$$

where $C_{2}$ is a constant independent of $n$.

Proposition 4.4. For $y \in C^{2 r}[0,1]$, we have

$$
\left\|\left(I-\pi_{n}\right) T\left(I-\pi_{n}\right) y\right\|_{\infty} \leqslant C_{3} h^{3 \tau}
$$

and

$$
\left\|T\left(I-\pi_{n}\right) T\left(I-\pi_{n}\right) y\right\|_{\infty} \leqslant C_{4} h^{4 r},
$$

where $C_{3}$ and $C_{4}$ are constants insependent of $n$. 
We now state our main result which follows immediately from Propositions (4.2) and (4.4).

THEOREM 4.5. Let $\pi_{n}$ be either the orthogonal projection or the interpolatory projection onto $X_{n}$. In the case of the orthogonal projection case we assume that $k(.,$. $\in C^{r}([0,1] \times[0,1])$ and $f \in C^{r}[0,1]$, while in the case of the interpolatory projection we assume that $k(.,.) \in C^{2 r}([0,1] \times[0,1])$ and $f \in C^{2 r}[0,1]$. Then

$$
\left\|u-u_{n}^{M}\right\|=O\left(h^{3 r}\right)
$$

and

$$
\left\|u-\widetilde{u}_{n}^{M}\right\|=O\left(h^{4 r}\right)
$$

ProOF: In the case of the orthogonal projections (4.12) follows from the estimate (3.1) of Theorem 3.1 and the estimate (4.5) of Proposition 4.2, while in the case of the interpolatory projections we use estimates (3.1) and (4.10) to deduce (4.13).

Since from (4.2) or (4.7) we have

$$
\begin{aligned}
\left\|\left(I-\pi_{n}\right) T u\right\|_{2} \leqslant\left\|\left(I-\pi_{n}\right) T u\right\|_{\infty} & \leqslant C_{1}\left\|(T u)^{(r)}\right\|_{\infty} h^{r} \\
& \leqslant C_{1}\|k\|_{r, \infty}\|u\|_{2} h^{r}
\end{aligned}
$$

it follows that

$$
\left\|\left(I-\pi_{n}\right) T\right\| \leqslant C_{1}\|k\|_{r, \infty} h^{r}
$$

We now deduce (4.13) from (3.2), (4.6), (4.12) and (4.14) or from (3.2), (4.11), (4.12) and (4.14).

REMARK 4.6. The above results should be compared with following known bounds.

$$
\begin{aligned}
\left\|u-u_{n}^{G}\right\| & =O\left(h^{r}\right) \\
\left\|u-u_{n}^{S}\right\| & =O\left(h^{2 r}\right) .
\end{aligned}
$$

REMARK 4.7. If the kernel is smooth as mentioned above, but the right hand side $f$ is less smooth, say $f \in C^{\rho}[0,1], \rho<r$, then from [14] we have

$$
\begin{aligned}
& \left\|u-u_{n}^{G}\right\|=O\left(h^{\rho}\right), \\
& \left\|u-u_{n}^{S}\right\|=O\left(h^{r+\rho}\right) .
\end{aligned}
$$

Consider the following Kantorovich method

$$
u_{n}^{K}-\pi_{n} T u_{n}^{K}=f
$$

and its iterated version

$$
\widetilde{u}_{n}^{K}=T u_{n}^{K}+f
$$


Then it is proved in [14] that

$$
\begin{aligned}
& \left\|u-u_{n}^{K}\right\|=O\left(h^{r}\right), \\
& \left\|u-\widetilde{u}_{n}^{K}\right\|=O\left(h^{2 r}\right) .
\end{aligned}
$$

It can be proved that

$$
\left\|u-u_{n}^{M}\right\|=O\left(h^{2 r+\rho}\right)
$$

and

$$
\left\|u-\widetilde{u}_{n}^{M}\right\|=O\left(h^{3 r+\rho}\right) .
$$

Thus when the kernel is smooth, irrespective of whether the right hand side $f$ has the same smoothness as the kernel or is less smooth, $u_{n}^{M}$ exhibits higher order of convergence as compared to $u_{n}^{G}, u_{n}^{S}, u_{n}^{K}$ and $\widetilde{u}_{n}^{K}$, whereas $\widetilde{u}_{n}^{M}$ has higher order of convergence than $u_{n}^{M}$.

REMARK 4.8. The full superconvergence order $4 r$ in the case when the right hand side is not smooth can be obtained by using a technique from [14]. The method proposed here is applied to the regularised equation

$$
v-T v=T f
$$

where $v=T u$. Thus let

$$
v_{n}^{M}-T_{n}^{M} v_{n}^{M}=T f
$$

and define

$$
\widetilde{v}_{n}^{M}=T v_{n}^{M}+T f
$$

Then

$$
\left\|v-\widetilde{v}_{n}^{M}\right\|=O\left(h^{4 r}\right)
$$

If we let

$$
\phi_{n}^{M}=\widetilde{v}_{n}^{M}+f
$$

then

$$
\left\|u-\phi_{n}^{M}\right\|=\left\|v-\widetilde{v}_{n}^{M}\right\|=O\left(h^{4 r}\right)
$$

\section{Numerical Results}

Consider the Fredholm integral equation of the second kind given by

$$
u(s)-\int_{0}^{1} \frac{1}{1+(s-t)^{2}} u(t) d t=1+(s)^{5 / 2} .
$$


The exact solution is not known, but it is clear that $u \in C^{2}[0,1]$, but $u$ does not belong to $C^{3}[0,1]$.

Let $X_{n}$ to be the space of piecewise constant functions $(r=1)$ with respect to the uniform partition

$$
0<\frac{1}{n}<\frac{2}{n}<\cdots<\frac{n}{n}=1 .
$$

and $\pi_{n}$ to be the interpolatory projection at the midpoints

$$
t_{k}^{(n)}=\frac{2 k-1}{n}, k=1, \ldots, n .
$$

In actual computations, the integral operator $T$ is replaced by $\bar{T}$ which is obtained by approximating the integral by the composite midpoint rule. It is given by

$$
\bar{T} x(s)=\sum_{k=1}^{m} \frac{1}{m}\left(\frac{1}{1+\left(s-t_{k}^{(m)}\right)^{2}}\right) x\left(t_{k}^{(m)}\right), s \in[0,1] .
$$

The solution obtained by using $\bar{T}$ instead of $T$ is denoted by $\bar{u}$.

For $m=512$, the quatities $\left|\left(\bar{u}-u_{n}^{G}\right)(s)\right|,\left|\left(\bar{u}-u_{n}^{S}\right)(s)\right|,\left|\left(\bar{u}-u_{n}^{M}\right)(s)\right|$ and $\left|\left(\bar{u}-\widetilde{u}_{n}^{M}\right)(s)\right|$ are computed at $s=1 / 512$ and $s=1 / 2-1 / 512$ for $n=4,8,16,32,64$ and 128 . Using two successive values of $n$, the orders of convergence are computed and are denoted by $\alpha, \beta, \gamma$ and $\delta$, respectively.

Table 5.1: $s=1 / 512$

\begin{tabular}{|l|c|c|c|c|c|c|c|c|}
\hline$n$ & Galerkin & $\alpha$ & Sloan & $\beta$ & New & $\gamma$ & Iterated New & $\delta$ \\
\hline 4 & $7.5 \times 10^{-1}$ & & $1.1 \times 10^{-1}$ & & $7.4 \times 10^{-4}$ & & $1.9 \times 10^{-5}$ & \\
8 & $3.5 \times 10^{-1}$ & 1.09 & $2.8 \times 10^{-2}$ & 2.03 & $7.8 \times 10^{-5}$ & 3.25 & $1.4 \times 10^{-6}$ & 3.73 \\
16 & $1.7 \times 10^{-1}$ & 1.06 & $7.1 \times 10^{-3}$ & 2.01 & $8.6 \times 10^{-6}$ & 3.19 & $9.3 \times 10^{-8}$ & 3.95 \\
32 & $8.0 \times 10^{-2}$ & 1.07 & $1.8 \times 10^{-3}$ & 2.01 & $9.7 \times 10^{-7}$ & 3.15 & $5.8 \times 10^{-9}$ & 4.00 \\
64 & $3.7 \times 10^{-2}$ & 1.11 & $4.4 \times 10^{-4}$ & 2.02 & $1.0 \times 10^{-7}$ & 3.17 & $3.6 \times 10^{-10}$ & 4.03 \\
128 & $1.6 \times 10^{-2}$ & 1.23 & $1.0 \times 10^{-4}$ & 2.07 & $1.1 \times 10^{-8}$ & 3.32 & $2.0 \times 10^{-11}$ & 4.14 \\
\hline
\end{tabular}

Table 5.2: $s=1 / 2-1 / 512$

\begin{tabular}{|l|c|c|c|c|c|c|c|c|}
\hline$n$ & Galerkin & $\alpha$ & Sloan & $\beta$ & New & $\gamma$ & Iterated New & $\delta$ \\
\hline 4 & $8.3 \times 10^{-2}$ & & $1.3 \times 10^{-1}$ & & $1.1 \times 10^{-3}$ & & $3.5 \times 10^{-6}$ & \\
8 & $5.2 \times 10^{-2}$ & 0.65 & $3.2 \times 10^{-2}$ & 2.03 & $1.3 \times 10^{-4}$ & 3.09 & $5.2 \times 10^{-7}$ & 2.76 \\
16 & $2.8 \times 10^{-2}$ & 0.89 & $7.9 \times 10^{-3}$ & 2.01 & $1.5 \times 10^{-5}$ & 3.07 & $3.7 \times 10^{-8}$ & 3.82 \\
32 & $1.4 \times 10^{-2}$ & 0.99 & $2.0 \times 10^{-3}$ & 2.01 & $1.8 \times 10^{-6}$ & 3.07 & $2.3 \times 10^{-9}$ & 3.97 \\
64 & $6.8 \times 10^{-3}$ & 1.07 & $4.9 \times 10^{-4}$ & 2.02 & $2.1 \times 10^{-7}$ & 3.12 & $1.4 \times 10^{-10}$ & 4.02 \\
128 & $2.9 \times 10^{-3}$ & 1.21 & $1.2 \times 10^{-4}$ & 2.07 & $2.1 \times 10^{-8}$ & 3.29 & $8.2 \times 10^{-12}$ & 4.13 \\
\hline
\end{tabular}

Since $r=1$, the expected values of $\alpha, \beta, \gamma$ and $\delta$ are $1,2,3$ and 4, respectively. It can be seen from the above tables that the computed values of $\alpha, \beta, \gamma$ and $\delta$ match 
well with the expected values. In addition, the error in the solution obtained by the iterated version of the method proposed here with $n=4$ is smaller than the error in the Galerkin/Sloan method with $n=128$.

\section{REFERENCES}

[1] G.A. Chandler, Superconvergence of numerical solutions of second kind integral equations, Ph.D. Thesis (Australian National University, ACT, Australia, 1979).

[2] F. Chatelin, Spectral approximation of linear operators (Academic Press, New York, 1983).

[3] F. Chatelin and R. Lebbar, 'The iterated projection solution for the Fredholm integral equation of second kind', J. Austral. Math. Soc. Ser. B 22 (1981), 439-451.

[4] C. de Boor, 'A bound on the $L_{\infty}$ norm of $L_{2}$-approximation by splines in terms of a global mesh ratio', Maths. Comput. 30 (1976), 765-771.

[5] C. de Boor and B. Swartz, 'Collocation at Gaussin points', SIAM J. Numer. Anal. 10 (1973), 582-606.

[6] J. Douglas, Jr., T. Dupont and L. Wahlbin, 'Optimal $L_{\infty}$ error estimates for Galerkin approximations to solutions of two point boundary value problems', Math. Comp. 29 (1975), 475-483.

[7] I.G. Graham, S. Joe and I.H. Sloan, 'Iterated Galerkin versus iterated collocation for integral equations of the second kind', IMA J. Numer. Anal. 5 (1985), 355-369.

[8] Q. Hu, 'Interpolation correction for collocation solutions of Fredholm integro-differential equations', Math. Comp. 67 (1998), 987-999.

[9] R.P. Kulkarni, 'A New Superconvergent projection method for approximate solutions of eigenvalue problems', Numer. Funct. Anal. Optim. 24 (2003), 75-84.

[10] Q. Lin, S. Zhang and N. Yan, 'An acceleration method for integral equations by using interpolation post-processing', Adv. Comput. Math. 9 (1998), 117-129.

[11] G.R. Richter, 'Superconvergence of piecewise polynomial Galerkin approximations for Fredholm integral equations of the second kind', Num. Math. 31 (1978), 63-70.

[12] E. Schock, 'Galerkin like methods for equations of the second kind', J. Integral Equations Appl. 4 (1982), 361-364.

[13] I.H. Sloan, 'Improvement by iteration for compact operator equations', Math. Comp. 30 (1976), 758-764.

[14] I.H. Sloan, 'Four variants of the Gałerkin method for Integral equations of the second kind', IMA J. Numer. Anal. 4 (1984), 9-17.

[15] A. Spence and K.S. Thomas, 'On superconvergence properties of Galerkin's method for compact operator equations', IMA J. Numer. Anal. 3 (1983), 253-271.

Department of Mathematics

Indian Institute of Technology, Bombay

Powai, Mumbai 400076

India

e-mail: rpk@math.iitb.ac.in 